науч. тр. Саратов: Изд-во Сарат. ун-та, 2011. Вып. 13. тода крупных частиц на устойчивость // ДифференC. 161-164.

3. Поттер Д. Вычислительные методы в физике. М. : к аэродинамике и теории вероятностей : межвуз. наМир, 1975. 392 с.

4. Шевырёв С. П. Исследование двумерной схемы ме-

\title{
Simulation of Incompressible Nonviscoussevere Fluid on a Regular Grid in Three-dimensional Space
}

\section{I. Liverovskiy, S. P. Shevirev}

Saratov State University, 83, Astrakhanskaya str., Saratov, 410012, Russia, LiverovskiyDI@gmail.com, ShevSP@ rambler.ru

This study focuses on modification of the method of Davydov (large particles) in the case of incompressible liquid. We consider the simulation of a heavy incompressible nonviscous fluid by Davydov's modified method in a three-dimensional case. Besides, comparison of the received results with a two-dimensional case is carried out. Formulas of modified Davydovs's method for the case of three spatial dimensions are lead out including difference analogue of the three-dimensional Poisson equation for the pressure. The criterion of stability is generalized. The description of the algorithm for constructing a three-dimensional free surface is provided. Implemented a three-dimensional computer graphics, showing development processes in online mode.

Key words: Davydov's method, fluid flow, method of large particles, free surface, Poisson equation for the pressure, the criterion of stability of difference schemes, symmetric flows.

\section{References}

1. Belotserkovskii O. M., Davydov Yu. M. Metod krupnykh chastits o gazovoi dinamike. Vychislitel'nyi eksperiment [Method of coarse particles in gas dynamics]. Moscow, Nauka, 1972, 392 p. (in Russian). 2. Liverovskiy D. I., Shevirev S. P. Metod Davydova dlia sluchaia neszhimaemoi neviazkoi tiazheloi zhidkosti na reguliarnoi setke [Davydov's method for a case of incompressible nonviscous heavy liquid on a regular grid]. Matematika. Mekhanika: sb. nauch. trudov, Saratov, Saratov Univ. Press, 2011, iss. 13, pp. 161-164 (in Russian).

3. Potter D. Computational Physics. New York, John Wiley and Sons, 1973.

4. Shevirev S. P. Issledovanie dvumernoi skhemy metoda krupnykh chastits na ustoichivost' [Research of the twodimensional scheme of a method of large particles on stability]. Differentsial'nye uravneniia $i$ teoriia funktsii $\checkmark$ prilozhenii $k$ aerodinamike $i$ teorii veroiatnostei: mezhouz. nauch. $s b$. [Differential equations and the theory of functions in the Annex to aerodynamics and the theory of probability: a collection of papers]. Saratov, Saratov Univ. Press, 1987, iss. 7, pp. 16-21 (in Russian).

УДК 519.6, 531

\section{ПРИМЕНЕНИЕ МЕТОДА ГАЛЁРКИНА К РЕШЕНИЮ ЛИНЕЙНЫХ ЗАДАЧ ОПТИМАЛЬНОГО УПРАВЛЕНИЯ}

\section{И. А. Панкратов}

Кандидат технических наук, доцент кафредры математического и компьютерного моделирования, Саратовский государственный университет им. Н. Г. Чернышевского, PankratovlA@info.sgu.ru

Рассмотрена линейная задача оптимального управления для случая, когда время окончания управляемого процесса фиксировано. Функционал, определяющий качество процесса управления, характеризует затраты энергии на управление. Предложен способ построения приближённого решения задачи, основанный на методе Галёркина. Приведены примеры численного решения задачи.

Ключевые слова: оптимальное управление, линейная система, метод Галёркина.

\section{1. ПОСТАНОВКА ЗАДАЧИ}

Рассмотрим управляемую систему, описываемую линейным векторным обыкновенным дифференциальным уравнением:

$$
\frac{d \boldsymbol{x}}{d t}=A \boldsymbol{x}+B u,
$$


где $\boldsymbol{x}, A, B$ - матрицы следующего вида:

$$
\boldsymbol{x}=\left(\begin{array}{c}
x_{1} \\
\vdots \\
x_{n}
\end{array}\right), \quad A=\left(\begin{array}{ccc}
A_{11} & \ldots & A_{1 n} \\
\vdots & \ddots & \vdots \\
A_{n 1} & \ldots & A_{n n}
\end{array}\right), \quad B=\left(\begin{array}{c}
B_{1} \\
\vdots \\
B_{n}
\end{array}\right),
$$

а управление $u$ есть скалярная функция, на которую не наложены ограничения.

Требуется перевести систему из начального положения при $t=0$ :

$$
x=x^{0}
$$

в конечное при $t=T$ :

$$
x=x^{\mathrm{K}} .
$$

При этом необходимо минимизировать функционал

$$
J=\int_{0}^{T} u^{2} d t
$$

Отметим, что минимум функционала (4) соответствует минимуму затрат энергии на управление, а время окончания управляемого процесса $T$ считается заданным.

Поставленная задача решается с помощью принципа максимума Понтрягина [1]. Введём вектор сопряжённых переменных $\boldsymbol{\psi}=\left(\psi_{1}, \ldots, \psi_{n}\right)^{T}$. Составим функцию Гамильтона - Понтрягина:

$$
H=-u^{2}+\sum_{j=1}^{n} \sum_{k=1}^{n} \psi_{j} A_{j k} x_{k}+\sum_{j=1}^{n} \psi_{j} B_{j} u
$$

Легко видеть [2], что сопряжённая система имеет вид

$$
\frac{d \boldsymbol{\psi}}{d t}=-A^{T} \boldsymbol{\psi}
$$

При этом оптимальное управление имеет вид

$$
u^{o p t}=\frac{1}{2} \sum_{j=1}^{n} B_{j} \psi_{j}
$$

Таким образом, задача сведена к краевой задаче с закреплённым правым концом траектории, описываемой системой линейных дифференциальных уравнений (1), (5), (6), порядка $2 n$ и $2 n$ краевыми условиями (2), (3).

\section{2. МЕТОД ВЗВЕШЕННЫХ НЕВЯЗОК}

Традиционно для решения задач оптимального управления применяются метод Ньютона, метод градиентного спуска [3] и др. Численному решению задач оптимального управления посвящены, например, работы Р. П. Федоренко [4], Ф. П. Васильева [5]. В общем случае отсутствуют формулы для нахождения неизвестных начальных значений сопряжённых переменных. Следует отметить также плохую сходимость начальных приближений для значений сопряжённых переменных к тем значениям, которые доставляют нули функциям невязок из-за постоянного попадания в их локальные минимумы, где ни метод Ньютона, ни метод градиентного спуска не дают хороших результатов. В настоящей работе предлагается искать приближённое решение рассматриваемой задачи оптимального управления в следующем виде [6]:

$$
\begin{gathered}
x_{j} \approx \hat{x}_{j}=x_{j}^{0}+\sum_{k=1}^{M} a_{j, k} N_{j, k}(t), \\
\psi_{j} \approx \hat{\psi}_{j}=\sum_{k=1}^{M} a_{n+j, k} N_{n+j, k}(t), \quad j=\overline{1, n} .
\end{gathered}
$$


Здесь $N_{j, k}(t), j=\overline{1,2 n}, k=\overline{1, M}$ - система линейно независимых базисных функций, удовлетворяющих следующим условиям:

$$
\begin{gathered}
N_{j, k}(0)=0, \quad j=\overline{1, n}, \quad k=\overline{1, M}, \\
\forall j=\overline{1, n}, \quad \exists k, l, m \quad N_{j, k}(T) \neq 0, \quad N_{n+j, l}(0) \neq 0, \quad N_{n+j, m}(T) \neq 0 .
\end{gathered}
$$

Из условий (8) следует, что

$$
\hat{x}_{j}(0)=x_{j}^{0}+\sum_{k=1}^{M} a_{j, k} N_{j, k}(0)=x_{j}^{0}, \quad j=\overline{1, n},
$$

и значит, функции $\hat{x}_{j}, j=\overline{1, n}$ автоматически удовлетворяют краевым условиям (2) при произвольных коэффициентах $a_{j, k}, j=\overline{1, n}, k=\overline{1, M}$.

Если выбранные функции $N_{j, k}, j=\overline{1,2 n}, k=\overline{1, M}$ непрерывны при $t \in[0 ; T]$ и все их производные существуют, то непосредственным дифференцированием (7) можно получить аппроксимации производных от $x_{j}$ и $\psi_{j}, j=\overline{1, n}$ :

$$
\begin{gathered}
\frac{d x_{j}}{d t} \approx \frac{d \hat{x}_{j}}{d t}=\sum_{k=1}^{M} a_{j, k} \frac{d N_{j, k}(t)}{d t}, \\
\frac{d \psi_{j}}{d t} \approx \frac{d \hat{\psi}_{j}}{d t}=\sum_{k=1}^{M} a_{n+j, k} \frac{d N_{n+j, k}(t)}{d t}, \quad j=\overline{1, n} .
\end{gathered}
$$

В скалярном виде фазовые и сопряжённые уравнения с учётом выражения (6) для оптимального управления запишутся так:

$$
\begin{gathered}
\frac{d x_{i}}{d t}=\sum_{j=1}^{n} A_{i j} x_{j}+\frac{1}{2} B_{i} \sum_{j=1}^{n} B_{j} \psi_{j}, \quad i=\overline{1, n} \\
\frac{d \psi_{i}}{d t}=-\sum_{j=1}^{n} A_{j i} \psi_{j}, \quad i=\overline{1, n} .
\end{gathered}
$$

Подставляя разложения (7) с учётом (9) в уравнения (10) и (11), получим невязки $R_{[0 ; T]}^{x_{i}}$ и $R_{[0 ; T]}^{\psi_{i}}$ следующего вида:

$$
\begin{gathered}
R_{[0 ; T]}^{x_{i}}=\sum_{k=1}^{M}\left[a_{i, k} \frac{d N_{i, k}}{d t}-\sum_{j=1}^{n} a_{j, k} A_{i j} N_{j, k}-\frac{1}{2} \sum_{j=1}^{n} a_{n+j, k} B_{i} B_{j} N_{n+j, k}\right]-\sum_{j=1}^{n} A_{i j} x_{j}^{0}, \quad i=\overline{1, n}, \\
R_{[0 ; T]}^{\psi_{i}}=\sum_{k=1}^{M}\left[a_{n+i, k} \frac{d N_{n+i, k}}{d t}+\sum_{j=1}^{n} a_{n+j, k} A_{j i} N_{j, k}\right], \quad i=\overline{1, n} .
\end{gathered}
$$

Для получения приближённых равенств $R_{[0 ; T]}^{x_{i}}=0$ и $R_{[0 ; T]}^{\psi_{i}}=0$ при $t \in[0 ; T]$ воспользуемся методом Галёркина [6], выбрав систему весовых функций $W_{s, k}=N_{s, k}, s=\overline{1,2 n}, k=\overline{1, M}$, и требуя, чтобы выполнялись равенства

$$
\begin{gathered}
\int_{0}^{T} R_{[0 ; T]}^{x_{s}} W_{s, k} d t=\int_{0}^{T} R_{[0 ; T]}^{x_{s}} N_{s, k} d t=0, \quad s=\overline{1, n}, \quad k=\overline{1, M}, \\
\int_{0}^{T} R_{[0 ; T]}^{\psi_{s-n}} W_{s, k} d t=\int_{0}^{T} R_{[0 ; T]}^{\psi_{s-n}} N_{s, k} d t=0, \quad s=\overline{n+1,2 n}, \quad k=\overline{1, M} .
\end{gathered}
$$

Так как разложения (7) не удовлетворяют априори граничным условиям (3), то положим

$$
\int_{0}^{T} R_{[0 ; T]}^{x_{s}} N_{s, k} d t=0, \quad s=\overline{1, n}, \quad k=\overline{1, M},
$$




$$
\int_{0}^{T} R_{[0 ; T]}^{\psi_{s-n}} N_{s, k} d t+\left.\left(\hat{x}_{s-n}-x_{s-n}^{\mathrm{K}}\right) \widetilde{W}_{s, k}\right|_{t=T}=0, \quad s=\overline{n+1,2 n}, \quad k=\overline{1, M}
$$

В общем случае весовые функции $W_{s, k}$ и $\widetilde{W}_{s, k}$ могут быть выбраны независимо, но из результатов численного решения задачи следует, что удобно взять $\widetilde{W}_{s, k}=-N_{s, k}, s=\overline{n+1,2 n}, k=\overline{1, M}$. Отметим также, что при аппроксимации различных фазовых и сопряжённых переменных можно использовать одинаковые базисные функции:

$$
N_{1, k}=N_{2, k}=\cdots=N_{n, k}=N_{k}^{x}, \quad N_{n+1, k}=N_{n+2, k}=\cdots=N_{2 n, k}=N_{k}^{\psi}, \quad k=\overline{1, M} .
$$

Уравнения (13) с учётом (7) можно записать так

$$
\int_{0}^{T} R_{[0 ; T]}^{\psi_{s-n}} N_{s, k} d t+\left(x_{s-n}^{0}+\sum_{k=1}^{M} a_{s-n, k} N_{s-n, k}(T)-x_{s-n}^{\mathrm{K}}\right) \widetilde{W}_{s, k}(T)=0,
$$

где $s=\overline{n+1,2 n}, k=\overline{1, M}$.

Соотношения (12), (14) представляют собой систему $2 M n$ линейных алгебраических уравнений относительно такого же числа неизвестных. Решив её, мы определим коэффициенты $a_{j, k}$ и тем самым закончим процесс построения приближённого решения уравнений (1), (5), удовлетворяющих условиям (2) и (3).

В частности, если

$$
N_{k}^{x}=t^{k}, \quad N_{k}^{\psi}=t^{k-1}, \quad k=\overline{1, M},
$$

то уравнения (12), (14) примут вид

$$
\begin{gathered}
\int_{0}^{T} \sum_{k=1}^{M}\left[a_{i, k} k t^{k-1}-\sum_{j=1}^{n} a_{j, k} A_{i j} t^{k}-\frac{1}{2} \sum_{j=1}^{n} a_{n+j, k} B_{i} B_{j} t^{k-1}\right] t^{s} d t=\int_{0}^{T} \sum_{j=1}^{n} A_{i j} x_{j}^{0} t^{s} d t, \\
\int_{0}^{T} \sum_{k=1}^{M}\left[a_{n+i, k}(k-1) t^{k-2}+\sum_{j=1}^{n} a_{n+j, k} A_{j i} t^{k-1}\right] t^{s-1} d t-\sum_{k=1}^{M} a_{i, k} T^{s+k-1}=\left(x_{i}^{0}-x_{i}^{\mathrm{K}}\right) T^{s-1} .
\end{gathered}
$$

Интегралы, входящие в систему (16), легко берутся. При этом уравнения (16) принимают вид

$$
\begin{gathered}
\sum_{k=1}^{M}\left[a_{i, k} \frac{k T^{s+k}}{s+k}-\sum_{j=1}^{n} a_{j, k} A_{i j} \frac{T^{s+k+1}}{s+k+1}-\frac{1}{2} \sum_{j=1}^{n} a_{n+j, k} B_{i} B_{j} \frac{T^{s+k}}{s+k}\right]=\sum_{j=1}^{n} A_{i j} x_{j}^{0} \frac{T^{s+1}}{s+1}, \\
\sum_{k=1}^{M}\left[a_{n+i, k} \frac{(k-1) T^{s+k-2}}{s+k-2}+\sum_{j=1}^{n} a_{n+j, k} A_{j i} \frac{T^{s+k-1}}{s+k-1}\right]-\sum_{k=1}^{M} a_{i, k} T^{s+k-1}=\left(x_{i}^{0}-x_{i}^{\mathrm{K}}\right) T^{s-1} .
\end{gathered}
$$

Отметим, что при $s=1$ и $k=1$ коэффициент при $a_{n+i, k}$ в уравнениях (17) должен равняться нулю.

Отметим также, что рассмотренный метод может применяться при наличии ограничения на управление и для решения нелинейных задач. При этом вместо (12), (13) нужно будет решать системы нелинейных алгебраических уравнений вида

$$
K(\mathbf{a}) \mathbf{a}=f
$$

или

$$
K \mathbf{a}=f(\mathbf{a}) .
$$

Здесь $K(\mathbf{a})$ и $f(\mathbf{a})$ - матрица жёсткости и столбец свободных членов соответственно, зависящие от вектора неизвестных а. Указанные системы нелинейных уравнений необходимо решать тем или иным итерационным методом (в качестве начального приближения удобно взять нулевой вектор). 


\section{3. ПРИМЕРЫ ЧИСЛЕННОГО РЕШЕНИЯ ЗАДАЧИ}

Пусть материальная точка массы $m$ кг движется прямолинейно под действием некоторой управляющей силы $F(t)$. Согласно второму закону Ньютона движение точки описывается уравнением

$$
m \frac{d^{2} x}{d t^{2}}=F(t)
$$

Введём фазовые координаты $x_{1}=x$ (координата точки), $x_{2}=d x / d t=v$ (скорость точки) и управляющий параметр $u=F(t) / m$. Тогда уравнение (20) можно представить в виде системы двух обыкновенных дифференциальных уравнений:

$$
\frac{d x_{1}}{d t}=x_{2}, \quad \frac{d x_{2}}{d t}=u .
$$

В начальный момент времени при $t=0$ состояние управляемой системы определяется соотношением

$$
\boldsymbol{x}=\boldsymbol{x}^{0}=\left(x_{1}^{0} ; x_{2}^{0}\right),
$$

в конечный момент времени

при $t=T$

$$
\boldsymbol{x}=\boldsymbol{x}^{\mathrm{K}}=\left(x_{1}^{\mathrm{K}} ; x_{2}^{\mathrm{K}}\right) .
$$

Положим, что функционал качества процесса управления имеет вид (4). Из уравнений (21) следует, что матрицы $A$ и $B$ имеют вид

$$
A=\left(\begin{array}{ll}
0 & 1 \\
0 & 0
\end{array}\right), \quad B=\left(\begin{array}{l}
0 \\
1
\end{array}\right) .
$$

Введём вектор сопряжённых переменных $\boldsymbol{\psi}=\left(\psi_{1}, \psi_{2}\right)^{T}$. Функция Гамильтона - Понтрягина примет вид

$$
H=-u^{2}+\psi_{1} x_{2}+\psi_{2} u .
$$

Система дифференциальных уравнений для сопряжённых переменных примет вид

$$
\frac{d \boldsymbol{\psi}}{d t}=-\left(\begin{array}{cc}
0 & 0 \\
-1 & 0
\end{array}\right) \boldsymbol{\psi}
$$

При этом оптимальное управление имеет вид

$$
u^{o p t}=\frac{\psi_{2}}{2} .
$$

Для решения системы (17) была составлена программа на языке $\mathrm{C}++$, реализующая метод Гаусса с выбором главного элемента по всей матрице.

На рис. $1, a-2$ приведены результаты решения задачи о движении точки под действием управляющей силы для следующих значений параметров: $M=3, T=4,\left(x_{1}^{0} ; x_{2}^{0}\right)=(1 ; 0),\left(x_{1}^{\mathrm{K}} ; x_{2}^{\mathrm{K}}\right)=(0 ; 0)$.

Отметим, что поставленную задачу можно решить аналитически. Легко видеть, что искомые функции $x_{1}(t), x_{2}(t), \psi_{1}(t)$ и $\psi_{2}(t)$ являются полиномами третьего, второго, нулевого и первого порядка соответственно. Поэтому результаты численного решения при трёх базисных функциях совпадают с аналитическим. Увеличение числа базисных функций в данном случае не приводит к уменьшению погрешности, так как неизвестные коэффициенты при базисных функциях $N_{4}^{x}, N_{4}^{\psi}, N_{5}^{x}, N_{5}^{\psi}$ и т. д. в разложениях (7) являются нулями.

Предположим теперь, что на материальную точку помимо управляющей силы действует сила сопротивления $F_{\text {сопр }}=-k v$. Тогда система двух обыкновенных дифференциальных уравнений, описывающих движение точки, примет вид

$$
\frac{d x_{1}}{d t}=x_{2}, \quad \frac{d x_{2}}{d t}=-\frac{k}{m} x_{2}+u .
$$

Из уравнений (23) следует, что матрицы $A$ и $B$ теперь имеют вид

$$
A=\left(\begin{array}{cc}
0 & 1 \\
0 & -k / m
\end{array}\right), \quad B=\left(\begin{array}{l}
0 \\
1
\end{array}\right) \text {. }
$$



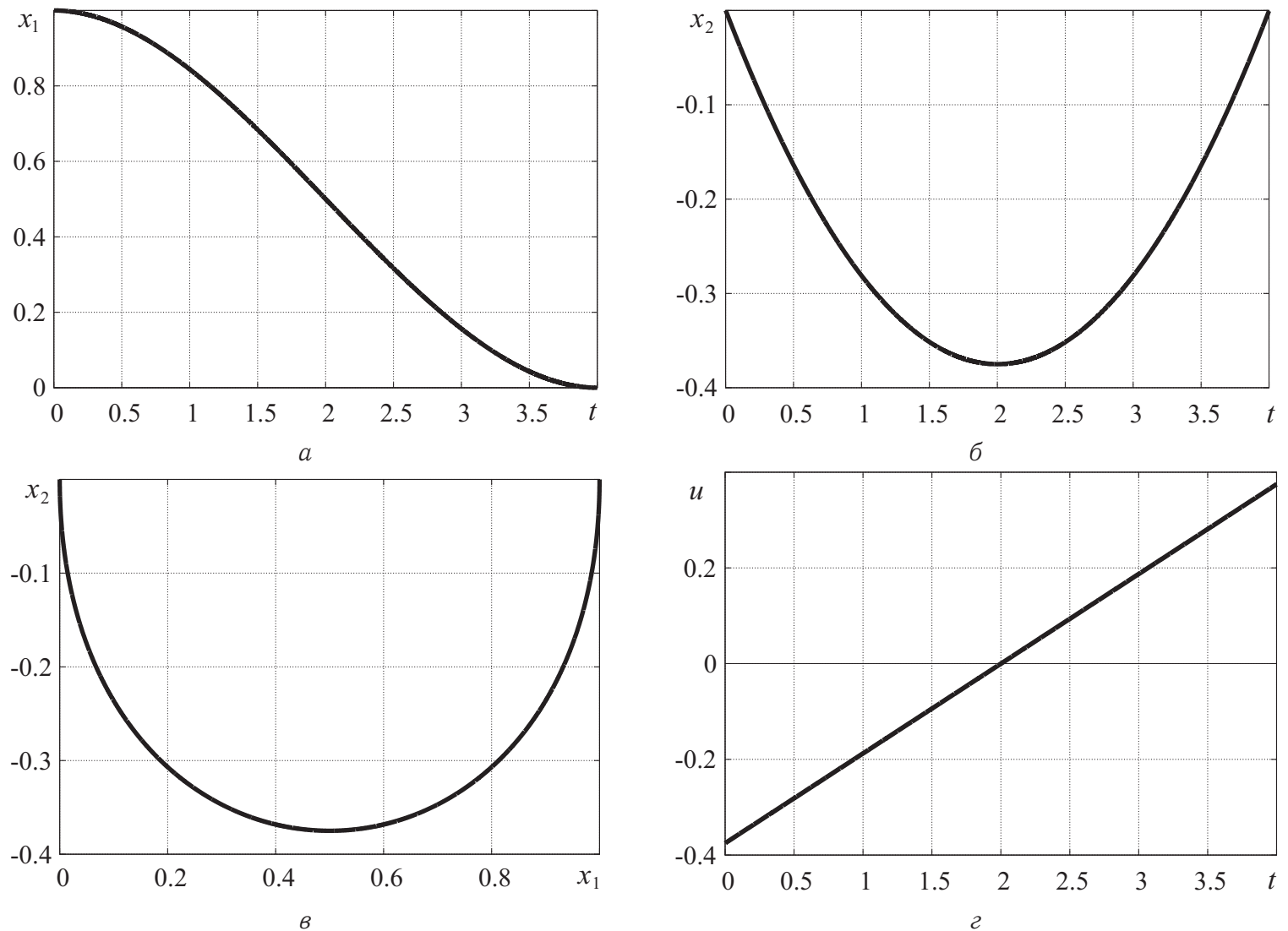

Рис. 1. Движение точки под действием управляющей силы $(M=3): a-$ координата точки; $\sigma-$ скорость точки; в - фазовый портрет; г-оптимальное управление

Соответственно система дифференциальных уравнений для сопряжённых переменных примет вид

$$
\frac{d \boldsymbol{\psi}}{d t}=-\left(\begin{array}{cc}
0 & 0 \\
-1 & k / m
\end{array}\right) \psi
$$

Оптимальное управление (22) не изменится.

На рис. 2 показаны результаты решения задачи о движении точки под действием управляющей силы и линейной силы сопротивления движению для следующих значений параметров: $M=9, T=4$, $k / m=1,\left(x_{1}^{0} ; x_{2}^{0}\right)=(1 ; 0),\left(x_{1}^{\mathrm{K}} ; x_{2}^{\mathrm{K}}\right)=(0 ; 0)$.
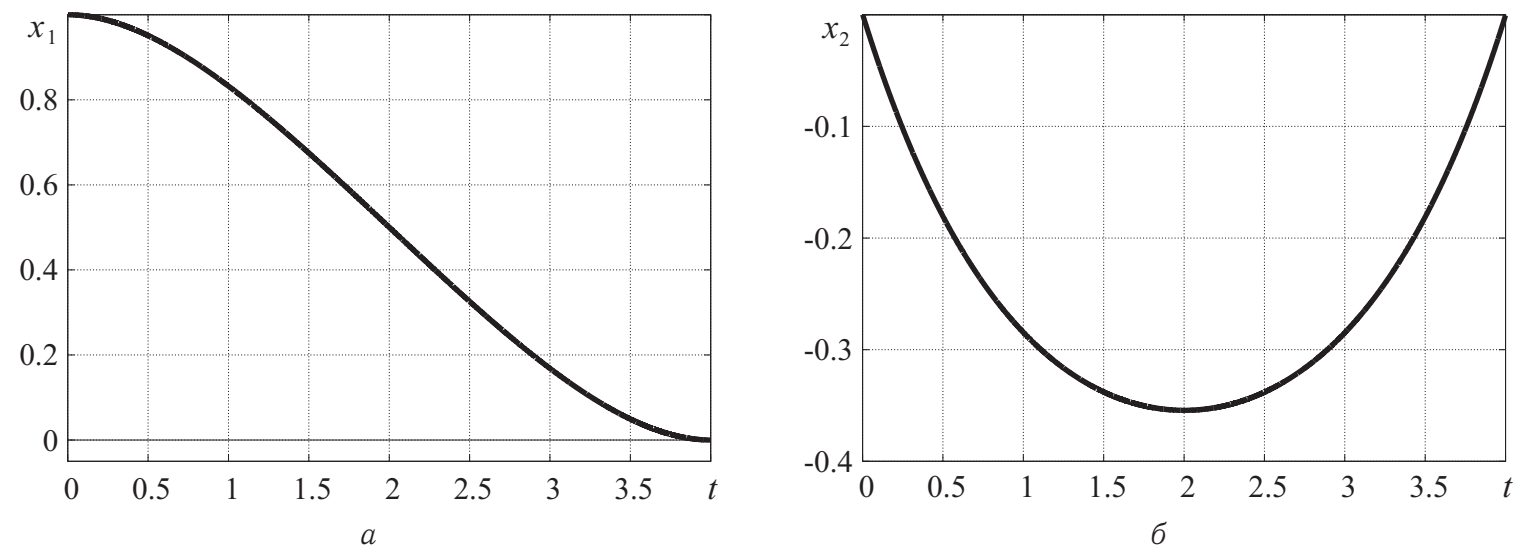

Рис. 2. Начало. Управляемое движение точки с учетом линейной силы сопротивления $(M=9)$ : $a$ - координата точки; $\sigma$ - скорость точки 

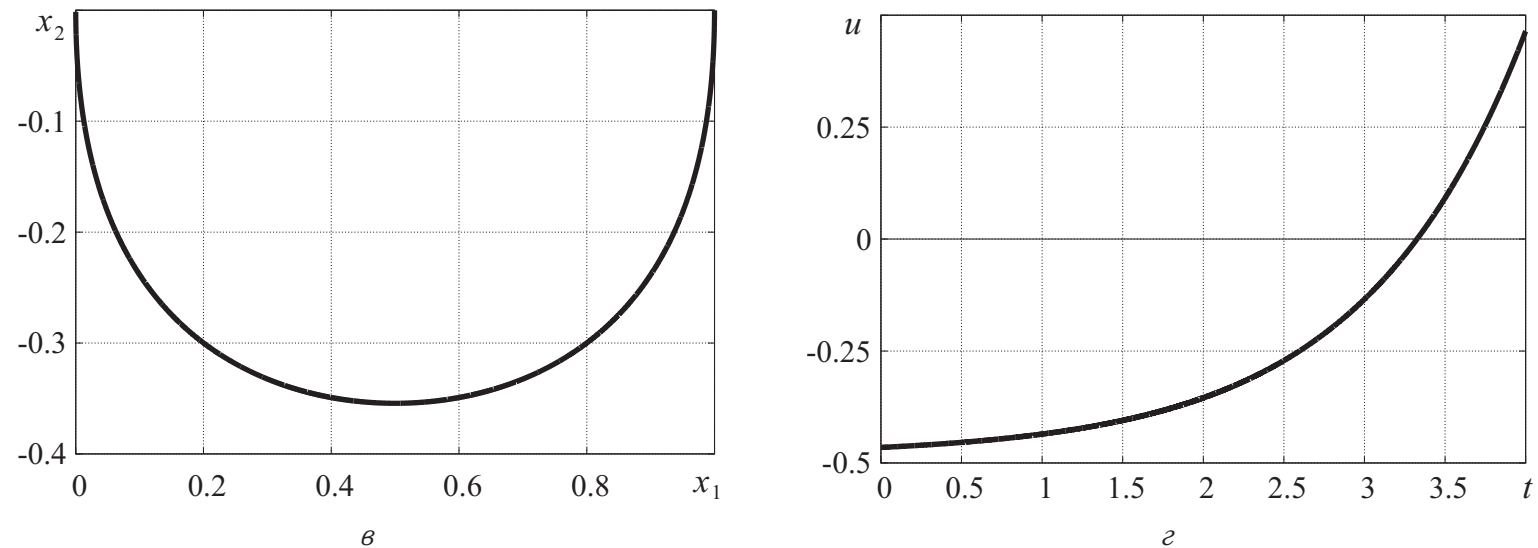

Рис. 2. Окончание: в - фазовый портрет; 2 - оптимальное управление

В таблице приведен закон изменения погрешности определения конечного положения точки в зависимости от числа степенных базисных функций (15) для тех же значений параметров задачи, что и на рис. 2.

Погрешность определения конечного положения точки

\begin{tabular}{|c|c|l|c|c|c|}
\hline$M$ & $x_{1}(T)$ & $x_{2}(T)$ & $\sqrt{x_{1}^{2}(T)+x_{2}^{2}(T)}$ & $\psi_{1}(0)$ & $\psi_{2}(0)$ \\
\hline 1 & 0 & -0.375 & 0.375 & -0.78125 & -0.6875 \\
\hline 2 & 0 & -0.25 & 0.25 & -0.78125 & -0.8125 \\
\hline 3 & $9.104 \cdot 10^{-15}$ & -0.1567398 & 0.1567398 & -0.8232759 & -0.7938871 \\
\hline 4 & $1.505 \cdot 10^{-13}$ & -0.0652635 & 0.0652635 & -0.9058572 & -0.8793439 \\
\hline 5 & $1.344 \cdot 10^{-11}$ & -0.0168948 & 0.0168948 & -0.9495609 & -0.9165632 \\
\hline 6 & $1.752 \cdot 10^{-11}$ & -0.0031820 & 0.0031820 & -0.9623113 & -0.9279307 \\
\hline 7 & $-8.443 \cdot 10^{-9}$ & -0.0004913 & 0.0004913 & -0.9648194 & -0.9301484 \\
\hline 8 & 0.0000001 & 0.0000654 & 0.0000654 & -0.9652157 & -0.9304994 \\
\hline 9 & -0.0000024 & -0.0000077 & 0.0000080 & -0.9652695 & -0.930547 \\
\hline
\end{tabular}

Значения сопряжённых переменных при $t=0$ (указанные в двух последних столбцах) могут быть использованы в качестве начального приближения при решении исходной задачи тем или иным итерационным методом. Отметим, что погрешность определения конечного значения координаты точки меньше, чем погрешность определения скорости точки в конце движения. Отметим также, что помимо (15) были рассмотрены следующие системы базисных функций (при этом особенно сложно подбирать подходящую систему базисных функций для сопряжённых переменных):

$$
N_{k}^{x}=(t / T)^{k}, \quad N_{k}^{\psi}=(t / T)^{k-1}, \quad k=\overline{1, M}
$$

и

$$
N_{k}^{x}=\sin k t, \quad N_{k}^{\psi}=t^{k-1}, \quad k=\overline{1, M} .
$$

Из результатов численного решения следует, что при использовании базисных функций (24) матрица жёсткости системы (12), (14) становится лучше обусловленной, чем при использовании системы (15). При $M \leq 5$ погрешность определения конечного положения точки меньше при использовании в качестве базисных функций $N_{k}^{x}=t^{k}$, а при $M>5$ меньшую погрешность дают базисные функции $N_{k}^{x}=\sin k t$.

Из результатов численного решения (см. рис. 1, 2) видно, что в первых двух рассмотренных примерах при $t=2$ скорость точки достигает своего минимального значения и $t=2$ - точка перегиба функции $x_{1}=x_{1}(t)$. 
Пусть на управление наложено ограничение вида

$$
-1 \leq u \leq 1
$$

Легко видеть, что оптимальное управление в задаче о движении материальной точки под действием управляющей силы и линейной силы сопротивления с учётом (25) принимает вид

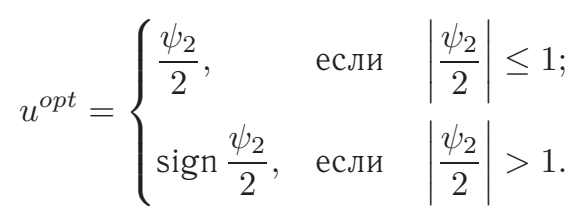

На рис. 3 приведены результаты решения задачи о движении точки под действием управляющей силы и линейной силы сопротивления движению при наличии ограничения (25) для следующих значений параметров: $M=9, T=7, k / m=1,\left(x_{1}^{0} ; x_{2}^{0}\right)=(1 ; 0),\left(x_{1}^{\mathrm{K}} ; x_{2}^{\mathrm{K}}\right)=(0 ; 0)$. Для решения системы уравнений вида (19) был применён метод простых итераций.
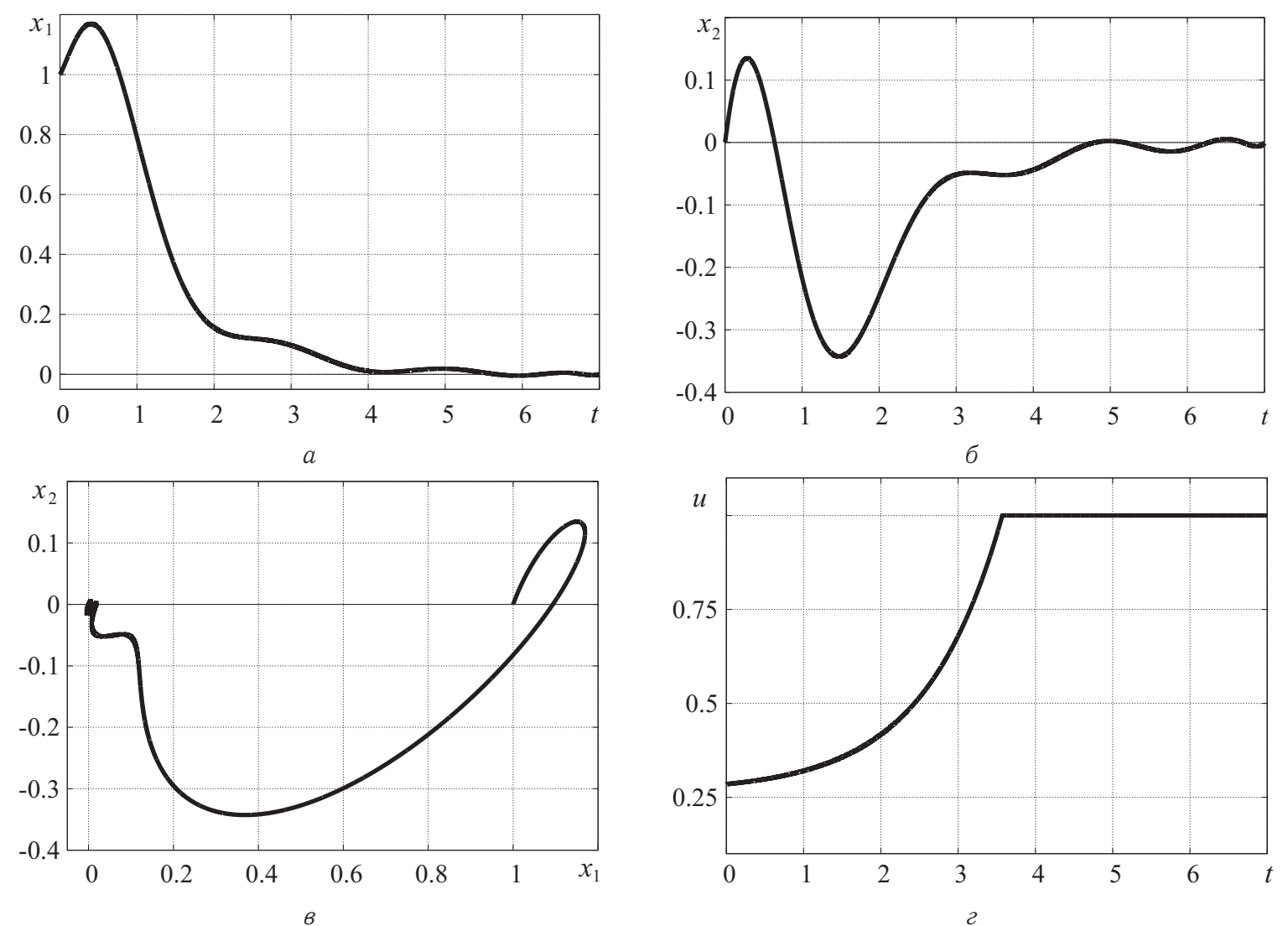

Рис. 3. Управляемое движение точки с учетом линейной силы сопротивления и ограничения (25) $(M=9)$ : $a$ - координата точки; $\sigma$ - скорость точки; в - фазовый портрет; г - оптимальное управление

Предположим теперь, что на материальную точку действуют управляющая сила $F(t)$ и нелинейная сила сопротивления $F_{\text {сопр }}=-k v^{2}$, а на управление $u$ ограничений не наложено.

Очевидно, что система фазовых уравнений в этом случае примет вид

$$
\frac{d x_{1}}{d t}=x_{2}, \quad \frac{d x_{2}}{d t}=-\frac{k}{m} x_{2}^{2}+u
$$

Легко видеть, что система сопряжённых уравнений будет иметь вид

$$
\frac{d \psi_{1}}{d t}=0, \quad \frac{d \psi_{2}}{d t}=-\psi_{1}+2 \frac{k}{m} x_{2} \psi_{2} .
$$

При этом оптимальное управление будет по-прежнему иметь вид (22). 
На рис. 4 приведены результаты решения задачи о движении точки под действием управляющей силы и квадратичной силы сопротивления движению для следующих значений параметров: $M=9$, $T=4, k / m=1,\left(x_{1}^{0} ; x_{2}^{0}\right)=(1 ; 0),\left(x_{1}^{\mathrm{K}} ; x_{2}^{\mathrm{K}}\right)=(0 ; 0)$. Для решения системы уравнений вида $(18)$ также был применён метод простых итераций.
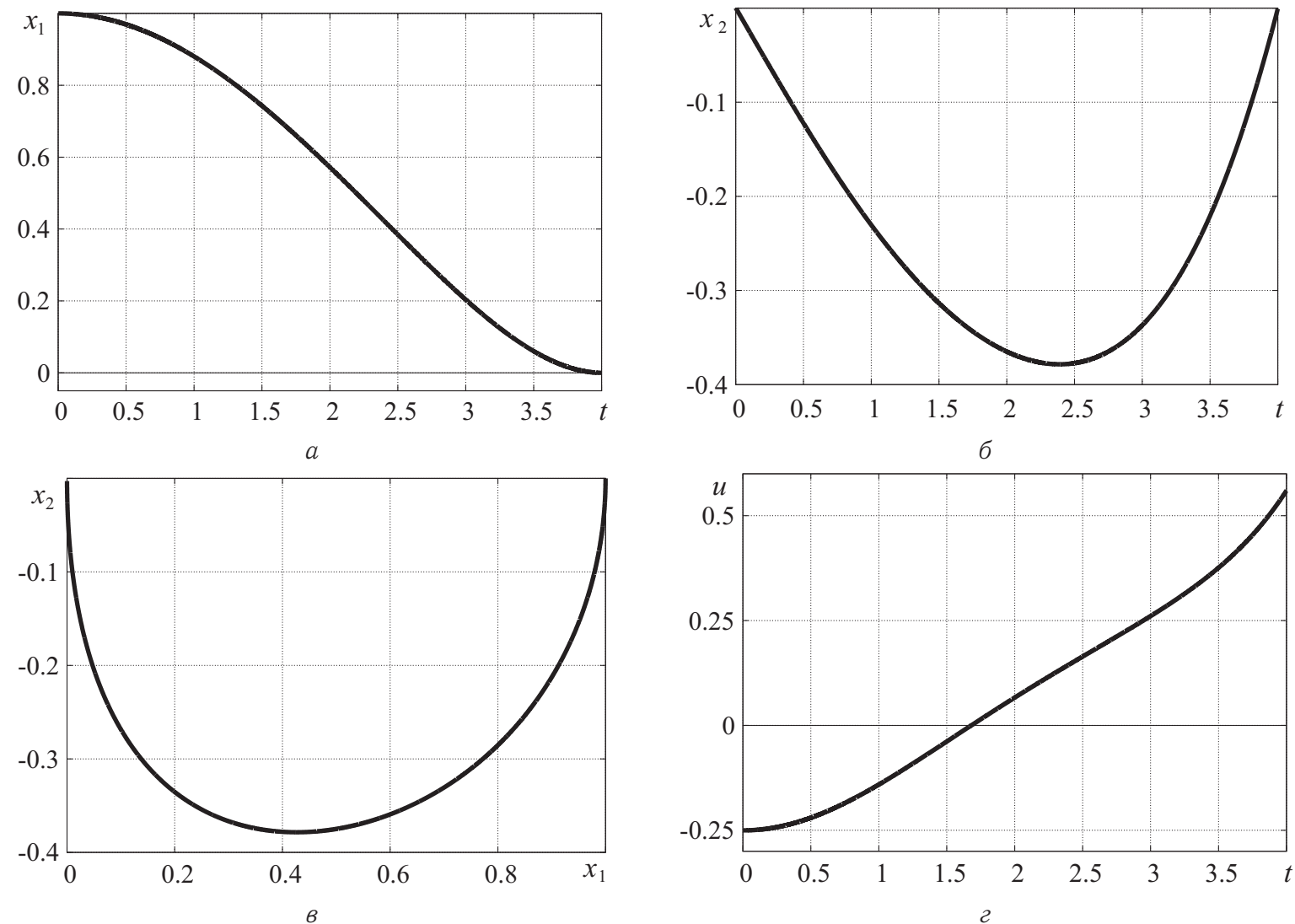

Рис. 4. Управляемое движение точки с учетом квадратичной силы сопротивления $(M=9): a-$ координата точки; $\sigma$ - скорость точки; в - фазовый портрет; 2 - оптимальное управление

В дальнейшем предполагается применить изложенный выше метод к решению задачи оптимальной переориентации орбиты космического аппарата под действием реактивной тяги, ортогональной плоскости орбиты [7-9].

Работа выполнена при финансовой поддержке РФФИ (проект № 12-01-00165-а).

\section{Библиографический список}

1. Понтрягин Л. С., Болтянский В. Г., Гамкрелидзе Р. В., Мищенко Е. Ф. Математическая теория оптимальных процессов. М. : Наука, 1983. 393 с.

2. Ройтенберг Я. Н. Автоматическое управление. М. : Наука, 1971. 396 с.

3. Моисеев Н. Н. Численные методы в теории оптимальных систем. М. : Наука, 1971. 424 с.

4. Федоренко Р. П. Приближенное решение задач оптимального управления. М. : Наука, 1978. 488 с.

5. Васильев Ф. П. Численные методы решения экстремальных задач. М. : Наука, 1988. 552 с.

6. Зенкевич O., Морган К. Конечные элементы и аппроксимация. М. : Мир, 1986. 318 с.

7. Панкратов И. А., Сапунков Я. Г., Челноков Ю. Н. Об одной задаче оптимальной переориентации орби- ты космического аппарата // Изв. Сарат. ун-та. Нов. сер. Сер. Математика. Механика. Информатика. 2012. Т. 12, вып. 3. С. 87-95.

8. Панкратов И. А., Сапунков Я. Г., Челноков Ю. Н. Решение задачи оптимальной переориентации орбиты космического аппарата с использованием кватернионных уравнений ориентации орбитальной системы координат // Изв. Сарат. ун-та. Нов. сер. Сер. Математика. Механика. Информатика. 2013. Т. 13, вып. 1, ч. 1. C. 84-92.

9. Челноков Ю. Н. Применение кватернионов в задачах оптимального управления движением центра масс космического аппарата в ньютоновском гравитационном поле. III // Космические исследования. 2003. Т. 41, вып. 5. С. 488-505. 


\title{
Using Galerkin Method for Solving Linear Optimal Control Problems
}

\begin{abstract}
I. A. Pankratov
Saratov State University, 83, Astrakhanskaya str., Saratov, 410012, Russia, PankratovlA@info.sgu.ru

The linear optimal control problem is considered. Duration of the controlled process is fixed. It is necessary to minimize the functional, that characterizes the energy consumption. A method of constructing an approximate solution based on the Galerkin method is proposed. Examples of numerical solutions of the problem are given.
\end{abstract}

Key words: optimal control, linear system, Galerkin method.

This work was supported by the Russian Foundation for Basic Research (project no. 12-01-00165-a).

\section{References}

1. Pontriagin L. S., Boltianskii V. G., Gamkrelidze R. V., Mishchenko E. F. Matematicheskaia teoriia optimal'nykh protsessov [The mathematical theory of optimal processes]. Moscow, Nauka, 1983, 393 p. (in Russian).

2. Roitenberg Ya. N. Avtomaticheskoe upravlenie [Automatic control]. Moscow, Nauka, 1983, 393 p. (in Russian).

3. Moiseev N. N. Chislennye metody v teorii optimal'nykh sistem [Numerical methods in the theory of optimal systems]. Moscow, Nauka, 1971, 424 p. (in Russian).

4. Fedorenko R. P. Priblizhennoe reshenie zadach optimal'nogo upravleniia [Approximate solution of optimal control problems]. Moscow, Nauka, 1978, 488 p. (in Russian).

5. Vasil'ev F. P. Chislennye metody resheniia ekstremal'nykh zadach [Numerical methods for solving extremal problems]. Moscow, Nauka, 1988, 552 p. (in Russian).

6. Zienkiewicz O., Morgan K. Finite elements and ap-

proximation. New York, Chichester, Brisbane, Toronto, John Wiley and Sons, 1983, 328 p.

7. Pankratov I. A., Sapunkov Ya. G., Chelnokov Yu. N. About a problem of spacecraft's orbit optimal reorientation. Izv. Saratov Univ. (N.S.), Ser. Math. Mech. Inform., 2012, vol. 12, iss. 3, pp. 87-95 (in Russian).

8. Pankratov I. A., Sapunkov Ya. G., Chelnokov Yu. N. Solution of a problem of spacecraft's orbit optimal reorientation using quaternion equations of orbital system of coordinates orientation. Izv. Saratov Univ. (N. S.), Ser. Math. Mech. Inform., 2013, vol. 13, iss. 1, pt. 1, pp. 87-95 (in Russian).

9. Chelnokov Yu. N. The use of quaternions in the optimal control problems of motion of the center of mass of a spacecraft in a newtonian gravitational field: III. Cosmic Research, 2003, vol. 41, no. 5, pp. 460-477. DOI 10.1023/A:1026098216710.

УДК 532.517.2:534.2

\section{ДВУХСЛОЙНОЕ ТЕЧЕНИЕ РАСПЛАВОВ ПОЛИМЕРОВ В КАНАЛАХ ФИЛЬЕР}

\author{
Б. А. Снигерев ${ }^{1}$, Ф. Х. Тазюков ${ }^{2}$ \\ ${ }^{1}$ Доктор технических наук, ведущий научный сотрудник лаборатории моделирования технологических процессов, Институт \\ механики и машиностроения Казанского научного центра РАН, snigerev@ mail.knc.ru \\ ${ }^{2}$ Доктор технических наук, профрессор кафредры теоретической механики и сопротивления материалов, Казанский нацио- \\ нальный исследовательский технологический университет, tazyukov@ mail.ru
}

Работа посвящена численному моделированию двухслойного течения нелинейно-вязких жидкостей в каналах фиильер. Движение жидкостей описывается уравнениями сохранения массы и импульса, дополненными реологическим уравнением состояния нелинейно-вязкой жидкости по модели Карро. Приводится методика численного решения задачи на основе метода конечных элементов. Исследована картина распределения скоростей жидкости, давления, напряжений, положения границы раздела в двухслойном потоке в зависимости от реологических свойств жидкости и режимов течения.

Ключевые слова: нелинейно-вязкая жидкость, граница раздела, двухслойное течение.

\section{ВВЕДЕНИЕ}

Слоистые, или стратифицированные, течения жидкостей давно привлекают внимание исследователей. Очень актуальными являются исследования, связанные с проблемами транспортировки сырой нефти по трубопроводам. Известны способы снижения давления, необходимого для перекачки нефти по трубам, путем добавления к нефти полимерного раствора, который оттесняясь к стенкам трубы, 